University of Nebraska - Lincoln

DigitalCommons@University of Nebraska - Lincoln

Faculty Publications: Department of

Entomology

Entomology, Department of

1997

\title{
Mitochondrial DNA Variation among Muscidifurax spp. (Hymenoptera: Pteromalidae), Pupal Parasitoids of Filth Flies (Diptera)
}

\author{
David B. Taylor \\ University of Nebraska-Lincoln, dave.taylor@ars.usda.gov \\ Richard D. Peterson II \\ University of Nebraska-Lincoln, padr20@yahoo.com \\ Allen L. Szalanski \\ University of Nebraska-Lincoln \\ James J. Petersen \\ University of Nebraska-Lincoln
}

Follow this and additional works at: https://digitalcommons.unl.edu/entomologyfacpub

Part of the Entomology Commons

Taylor, David B.; Peterson, Richard D. Il; Szalanski, Allen L.; and Petersen, James J., "Mitochondrial DNA Variation among Muscidifurax spp. (Hymenoptera: Pteromalidae), Pupal Parasitoids of Filth Flies (Diptera)" (1997). Faculty Publications: Department of Entomology. 206.

https://digitalcommons.unl.edu/entomologyfacpub/206

This Article is brought to you for free and open access by the Entomology, Department of at DigitalCommons@University of Nebraska - Lincoln. It has been accepted for inclusion in Faculty Publications: Department of Entomology by an authorized administrator of DigitalCommons@University of Nebraska - Lincoln. 


\title{
Mitochondrial DNA Variation Among Muscidifurax spp. (Hymenoptera: Pteromalidae), Pupal Parasitoids of Filth Flies (Diptera)
}

\author{
DAVID B. TAYLOR, RICHARD D. PETERSON II, ALLEN L. SZALANSKI, ${ }^{1}$ AND JAMES J. PETERSEN
}

Midwest Livestock Insects Research Laboratory, USDA-ARS, Department of Entomology, University of Nebraska, Lincoln, NE 68583

\begin{abstract}
Ann. Entomol. Soc. Am. 90(6): 814-824 (1997)
ABSTRACT Polymerase chain reaction-restriction fragment length polymorphism (PCRRFLP) and sequencing analyses were used to characterize an amplicon of $\approx 625$ bp in 4 of the 5 nominate species of Muscidifurax Girault \& Sanders, pupal parasitoids of muscoid flies. A single polymorphic nucleotide site was observed among 2 samples of $M$. raptor Girault \& Sanders. No sequence variation was observed among 3 samples of $M$. raptorellus Kogan \& Legner. The sequence of $M$. uniraptor Kogan \& Legner was identical to that of $M$. raptorellus. Nucleotide divergence among the Muscidifurax spp. ranged from 0.14 to 0.18 substitutions per nucleotide. Muscidifurax zaraptor Kogan \& Legner exhibited multiple haplotypes, 2 of which were characterized by sequencing and 4 others by PCR-RFLP. The sequenced haplotypes differed by 0.08 nucleotide substitutions per site. Restriction site analysis indicated that nucleotide divergence ranged from 0.03 to 0.10 among all 6 haplotypes. Analysis of progeny from individual females indicated that the observed variation in $M$. zaraptor was caused by multiple haplotypes within individuals rather than differentiation among individuals. These results bring to question the specific status of $M$. uniraptor and indicate that the genus is native to the Western Hemisphere, and not introduced with their primary host, Musca domestica L., as previously proposed. Heteroplasmy and translocation of a portion of the mitochondrial genome to the nuclear genome are discussed as possible causes for the variation observed in M. zaraptor.
\end{abstract}

KEY WORDS Muscidifurax, polymerase chain reaction-restriction fragment length polymorphism, mitochondrial DNA, phylogeny

WASPS IN THE genus Muscidifurax are pupal parasitoids of muscoid flies, especially the house fly, Musca domestica L., and the stable fly, Stomoxys calcitrans (L.), and are among the most promising biological control agents for these flies in the confined livestock environment (Miller and Rutz 1990, Petersen et al. 1990). Muscidifurax includes 5 species. Muscidifurax raptor Girault \& Sanders, the most widespread, is found throughout the temperate and semitropical regions of the world (Kogan and Legner 1970). The remaining 4 species are limited to the New World. Muscidifurax zaraptor Kogan \& Legner is found sympatrically with $M$. raptor in western North America (Kogan and Legner 1970, Lysyk 1995), M. raptoroides Kogan \& Legner and M. raptorellus Kogan \& Legner are found allopatrically in Central and South America, respectively (Kogan and Legner 1970). Two forms of M. raptorellus have been reported, one solitary and the other gregarious (Kogan and Legner 1970, Legner 1988). Muscidifurax uniraptor Kogan \& Legner is a parthenogenic

\footnotetext{
${ }^{1}$ Department of Plant Pathology, University of Nebraska, Lincoln, NE 68583.
}

species known only from the island of Puerto Rico (Kogan and Legner 1970).

The geographic origins and phylogenetic relationships of Muscidifurax are unclear. Kogan and Legner (1970) proposed 2 alternatives for the origins of the genus. First, they originated in the Ethiopian region and were introduced to the New World along with house flies, or 2nd, they are native to the New World and have secondarily adapted to house flies. Kogan and Legner conclude that the "remarkable preference of Muscidifurax spp. for house flies as compared to native Nearctic flies" indicates an Old World origin for the genus. Legner (1983) indicates that the dependence of Muscidifurax upon the "barnyard" environment outside of Africa is further evidence that the genus was not native to the New World. However, to accept an Old World origin of the genus and account for the 4 species endemic to the New World, one must accept a very rapid rate of speciation following their introduction.

Despite the interest in these species for biological control of filth flies and the questions concerning their geographic origin, little work has been done on the population genetics and genetic structure of Muscidifurax. Propp (1986) used allozymes to ex- 
Table 1. Strains of Muscidifurax spp. used in this study

\begin{tabular}{|c|c|c|c|}
\hline Species & Origin & $\begin{array}{l}\text { Date } \\
\text { collected }\end{array}$ & Source ${ }^{a}$ \\
\hline M. raptor & $\begin{array}{l}\text { Nebraska } \\
\text { New York }\end{array}$ & $\begin{array}{l}1995 \\
\text { Unknown }\end{array}$ & $\begin{array}{l}\text { MLIRL } \\
\text { Cornell University }\end{array}$ \\
\hline M. raptorellus & $\begin{array}{l}\text { Chile } \\
\text { Nebraska } \\
\text { Peru }\end{array}$ & $\begin{array}{l}\text { Unknown } \\
1991 \\
\text { Unknown }\end{array}$ & $\begin{array}{l}\text { Legner via MLIRL } \\
\text { MLIRL } \\
\text { Legner via MLIRL }\end{array}$ \\
\hline $\begin{array}{l}\text { M. uniraptor } \\
\text { M. zaraptor }\end{array}$ & $\begin{array}{l}\text { Puerto Rico } \\
\text { Nebraska } \\
\text { Nebraska }\end{array}$ & $\begin{array}{l}\text { Unknown } \\
1991-1993 \\
1995\end{array}$ & $\begin{array}{l}\text { Rochester University } \\
\text { MLIRL } \\
\text { MLIRL }\end{array}$ \\
\hline
\end{tabular}

${ }^{a}$ MLIRL, Midwest Livestock Insect Research Laboratory, USDA-ARS, Lincoln, NE; Legner via MLIRL, originally collected by E. F. Legner and transferred to MLIRL in 1989.

amine several populations of $M$. raptor and $M$. zaraptor. He found relatively low levels of differentiation among geographic isolates of the 2 species, but a high level of differentiation was observed between species. Antolin et al. (1996) used RAPD-PCR to examine 3 Muscidifurax spp. They were able to differentiate the species and associate the gregarious North American Muscidifurax sp. with M. raptorellus, but indicated they were unable to explore phylogenetic relationships because of the nature of the RAPD-PCR data.

The purpose of this study was to develop molecular diagnostic characters and examine genetic differentiation among Muscidifurax spp. A region of the mitochondrial genome, $\approx 625 \mathrm{bp}$, including parts of the cytochrome oxidase I (COI) and II (COII) genes as well as the entire tRNA leucine (tRNA ${ }^{\text {leu }}$ ) gene was examined by polymerase chain reactionrestriction fragment length polymorphism (PCRRFLP) and sequencing. Representative samples of 4 of the 5 species in the genus (samples of $M$. raptoroides were not available) were included in this study.

\section{Materials and Methods}

Samples. Muscidifurax samples were obtained from established colonies (Table 1). The Chile and Nebraska samples of $M$. raptorellus represented gregarious populations and the Peru sample was solitary. The Apis mellifera lingustica $\mathrm{L}$. control sample was collected from an apiary in Lincoln, NE, and the Cochliomyia macellaria (F.) samples were from a laboratory colony originating in Fargo, ND. Muscidifurax colonies were maintained on freeze-killed M. domestica pupae (Petersen and Matthews 1984).

Isofemale lines were initiated by isolating individual females (presumably mated) from the main colony and placing them in small plastic cups $(2 \mathrm{~cm}$ diameter, $2 \mathrm{~cm}$ high) with $\approx 150$ freeze-killed fly pupae. Progeny emerged in $3 \mathrm{wk}$. Specimens were stored at $-80^{\circ} \mathrm{C}$.

Voucher specimens from each of the Muscidifurax samples have been placed in the collection of the University of Nebraska State Museum, Lincoln.

DNA Extraction and Amplification. Pools of 5-25 wasps were used for each DNA extraction. DNA was

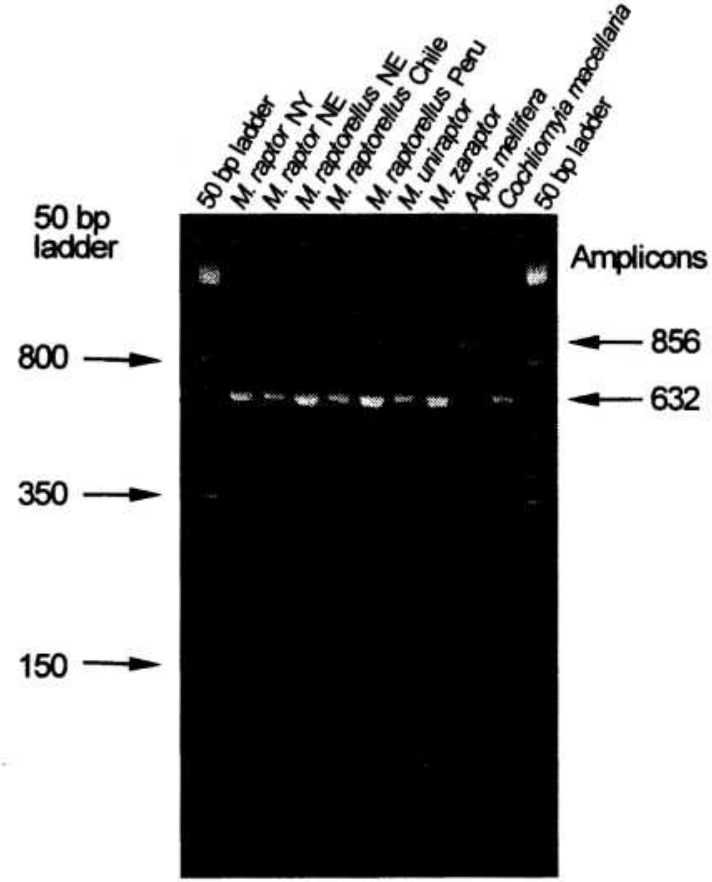

Fig. 1. Intact amplicon from 4 Muscidifurax spp., A mellifera, and C. macellaria on $2.5 \%$ MetaPhor agarose gel. Primers were 5' ATACCTCGACGTTATTCAGA $3^{\prime}$ and 5 ' TCAATATCATTGATGACCAAT 3 '.

extracted with the chloroform-phenol technique as outlined in Taylor et al. (1996).

A region of $\mathrm{mtDNA}, \approx 625 \mathrm{bp}$ long, was amplified using the primers 5' ATACCTCGACGTTATTCAGA $3^{\prime}$ (= S2792; Bogdanowicz et al. 1993) and 5' TCAATATCATTGATGACCAAT $3^{\prime}$ (K. Pruess, personal communication). The $5^{\prime}$ ends of these primers were located at bp 2773 and 3400 of the Drosophila yakuba mtDNA map (Clary and Wolstenholme 1985), respectively.

For amplification, $1 \mu \mathrm{l}$ of sample DNA was added to a reaction mixture containing $2.5 \mu \mathrm{l}$ of reaction buffer (Perkin-Elmer, Norwalk, CT), $2 \mu$ l of dNTP mix (10 mM each-dATP, dTTP, dCTP and dGTP), $1 \mu \mathrm{l}$ of each primer $(20 \mathrm{mM}), 1.0$ unit of Taq polymerase (Perkin-Elmer) and deionized water to a volume of $25 \mu \mathrm{l}$. Amplifications were done in a Perkin Elmer Cetus Model 9600 thermocycler programmed for 35 cycles of $92^{\circ} \mathrm{C}$ for $1 \mathrm{~min}, 55^{\circ} \mathrm{C}$ for 1 min, and $72^{\circ} \mathrm{C}$ for 1 or $2 \mathrm{~min}$. Amplification products were stored at $4^{\circ} \mathrm{C}$.

Restriction Endonuclease Digests. Twenty-seven restriction enzymes-Alu I, Apo I, Ase I, Ava I, Ban II, Bfa I, Bsr I, Dde I, Dpn II, Dra I, EcoR I, EcoR V, Hae III, Hinc II, Hind III, Hinf I, Hpa I, Mse I, Msp I, Pvu II, Rsa I, Sac I, Sau96 I, ScrF I, Ssp I, Taq I, and $\mathrm{Xba} \mathrm{I}$-were screened. Digests for polyacrylamide gel electrophoresis (PAGE) were done using $1.5 \mu \mathrm{l}$ of PCR product, $0.2 \mu$ l enzyme (New England Biolabs, Beverly, MA), $1 \times$ buffer (New England Bio- 

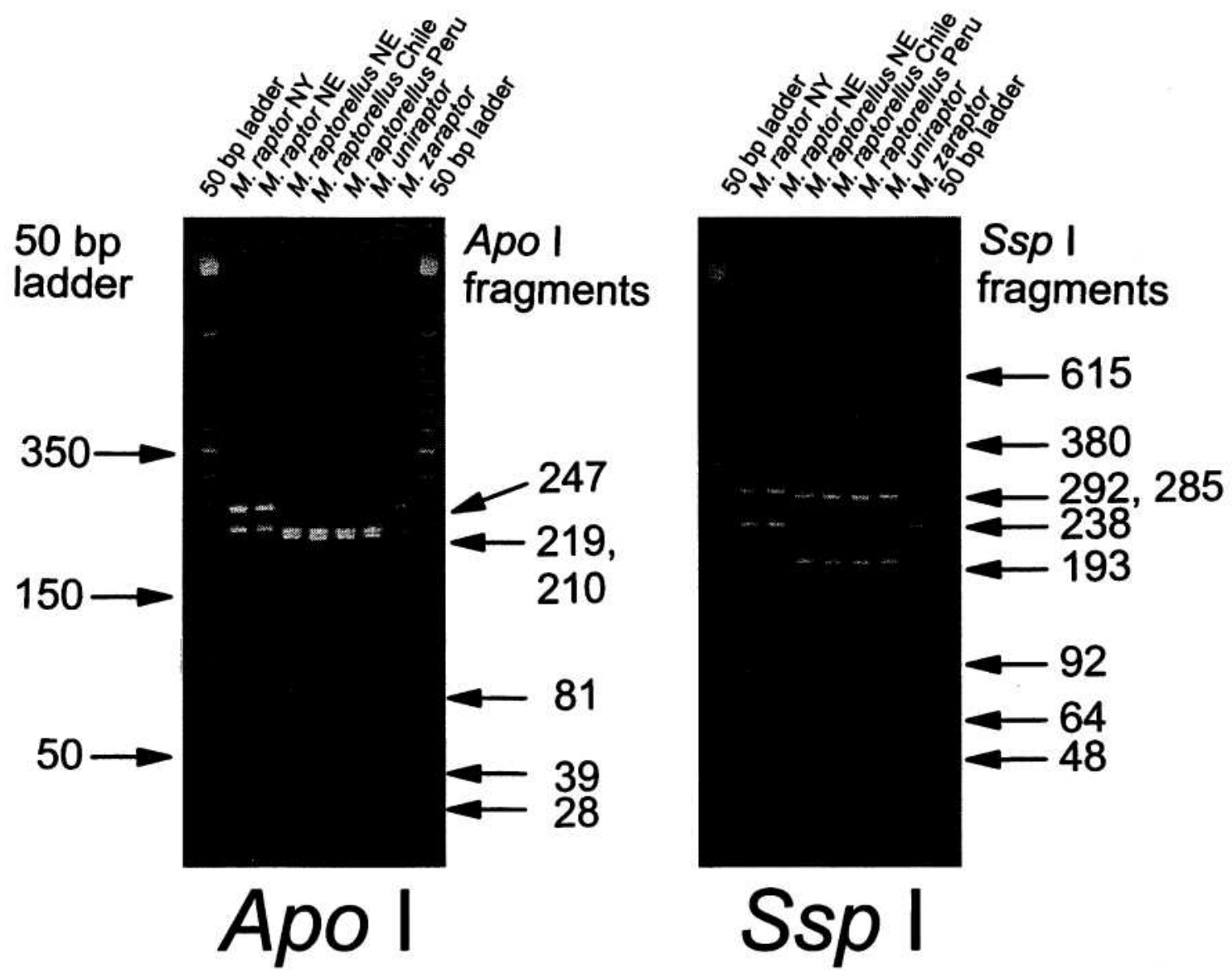

Fig. 2. Apo I and Ssp I digests of Muscidifurax spp. on 2.5\% MetaPhor agarose gels.

labs) and water to bring the volume to $5 \mu \mathrm{l}$ in $200-\mu \mathrm{l}$ tubes. For agarose gel electrophoresis, all quantities were increased 2.5 times. Samples were incubated at $37^{\circ} \mathrm{C}$ for $3-16 \mathrm{~h}$ and stored at $4^{\circ} \mathrm{C}$ until further analysis.

Electrophoresis. For PAGE, $1.5 \mu \mathrm{l}$ of loading buffer ( $10 \%$ Ficoll 400 [Sigma, St. Louis, MO], $0.25 \%$ Bromophenol Blue [Sigma], $50 \mathrm{mM}$ EDTA, $10 \mathrm{mM}$ Tris- $\mathrm{HCl} \mathrm{pH} 7.5$ ) was added to the 5- $\mu$ l digest product. The entire digest product $(6.5 \mu \mathrm{l})$ was loaded onto a $10 \%$ acrylamide gel $(1 \times$ Tris:borate:EDTA (TBE), 0.5\% Photoflo-200 [Kodak, Rochester, NY], $0.15 \%$ TEMED, and $0.05 \%$ ammonium persulfate). A molecular size standard, pGEM (Promega, Madison, WI), was included on each gel. Hoefer (Hoefer Scientific Instruments, San Francisco, CA) SE 600 electrophoresis units with gels $(16$ by $20 \mathrm{~cm}$ by 0.75 $\mathrm{mm}$ ) and 28 well combs were used for electrophoresis. Gels were run in $1 \times$ TBE buffer at a constant 300 $\mathrm{V}(15 \mathrm{~mA} / \mathrm{gel})$ for $1.5 \mathrm{~h}$ at $20^{\circ} \mathrm{C}$. Gels were stained

Table 2. Restrietion fragment lengths estimated on $2.5 \%$ Metaphor agarose gels for Muscidifurax species

\begin{tabular}{lllll}
\hline \hline $\begin{array}{l}\text { Restriction } \\
\text { enzymes }\end{array}$ & \multicolumn{1}{c}{ M. raptor NE } & \multicolumn{1}{c}{ M. raptorellus } & \multicolumn{1}{c}{ M. uniraptor } & M. zaraptor \\
\hline Apo I & $247,219,52,42,28$ & $219,210,81,39$ & $219,210,81,39$ & $247,219,210,189,98,40$ \\
Ase I & 327,297 & $387,165,59$ & $387,165,59$ & $454,234,180,167$ \\
Dpn II & 373,239 & 628 & 628 & 373,239 \\
Dra I & $321,243,65$ & $245,239,80,37,31$ & $245,239,80,37,31$ & $328,248,184,149,38,33,27$ \\
Hae III & 614 & 477,147 & 477,147 & 631 \\
Hinf I & 600,34 & $454,133,34,24$ & $454,133,34,24$ & $585,537,446,257,179,136,41,31$ \\
Mse I & $149,98,94,71,53,33,14$ & $147,92^{\circ}, 58,32,25,17$ & $147,92^{a}, 58,32,25,17$ & $149,117,69,36,27$ \\
Ssp I & $292,239,98$ & $285,193,64,48$ & $285,193,64,48$ & $615,380,329,287,270,236$, \\
& & & $222,190,184,99$ \\
Taq I & 452,162 & 618 & 618 & $463,411,336,295,164,108,93$ \\
\hline
\end{tabular}

a Possibly double bands. 
M. raptor

$M$. raptorelius

M. zaraptor I

M. zaraptor II

$M$. raptor

M. raptorellus

M. zaraptor I

M. zaraptor II

M. raptor

M. raptorellus

M. zaraptor I

M. zaraptor II

$M$. raptor

$M$. raptorellus

M. zaraptor I

M. zaraptor II

M. raptor

M. raptorellus

M. zaraptor I

M. zaraptor II

M. raptor

$M$. raptorellus 121

M. zaraptor I 117

M. zaraptor II 121

\section{M. raptor}

M. raptorellus

M. zaraptor I

M. zaraptor II

M. raptor 181

M. raptorelius 181

M. zaraptor I 177

M. zaraptor II 181

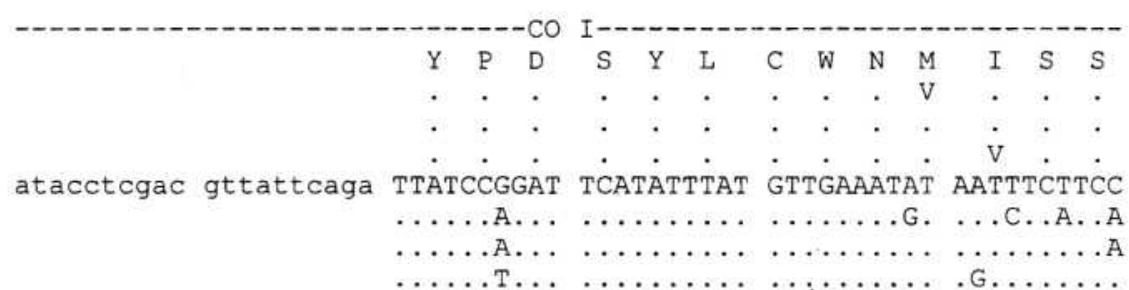

\begin{tabular}{|c|c|c|c|c|c|c|c|c|c|c|c|c|c|c|c|c|c|c|c|c|}
\hline L & G & S & $\mathrm{F}$ & I & S & $M$ & M & S & $\mathrm{T}$ & $M$ & L & $\mathrm{F}$ & $\mathrm{F}$ & F & I & I & & & $E$ & S \\
\hline 9 & . & . & I & . & . & . & $\therefore$ & . & & L & $\mathrm{F}$ & . & . & . & . & . & & & . & \\
\hline & . & . & I & . & . & . & V & . & & I & $\mathrm{F}$ & . & & L & L & & $\mathrm{F}$ & $E$ & K & \\
\hline & . & . & I & . & . & . & V & . & & I & $\mathrm{F}$ & . & . & $Y$ & & . & & & & \\
\hline
\end{tabular}

61 CTGGGAAGAT TTATTTCTAT AATAAGAACA ATATTATTTT TCTTCATTAT TTGAGAAAGA

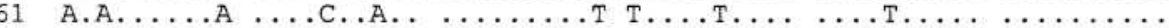

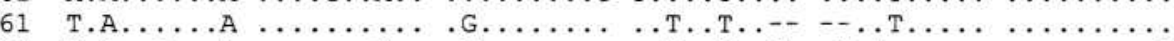

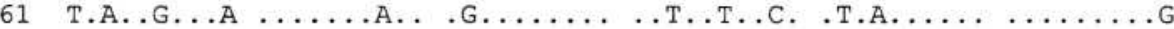

$$
\text { 西 }
$$

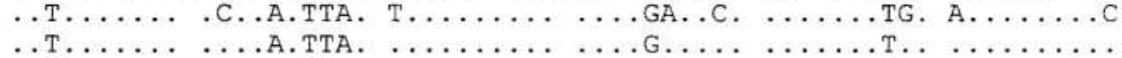

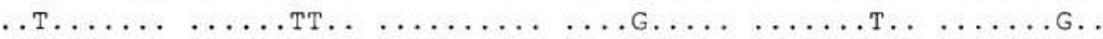

\begin{tabular}{|c|c|c|c|c|c|c|c|c|c|c|c|c|c|c|c|c|c|c|}
\hline M & S & F & P & P & $S$ & F & $\mathrm{H}$ & s & E & S & E & I & P & K & I & Y & K & * \\
\hline . & A & . & . & . & . & . & . & . & . & . & . & . & . & . & . & E & $\mathrm{N}$ & * \\
\hline$\dot{x}$ & $\mathrm{~N}$ & $\dot{1}$ & $\dot{x}$ & · & $\dot{x}$ & M & . & $\dot{8}$ & $\dot{0}$ & $\mathrm{~N}$ & $\therefore$ & $\dot{0}$ & . & $\therefore$ & L & $\therefore$ & Y & $*$ \\
\hline ATA & $\mathrm{TCT}$ & TTTC & $\mathrm{CAC}$ & CCT" & TCA: & & TCATT & TCAT & TTT & AGAG & AAA & ATTC & & AAAA & ATT & TA & TAAAI & TAATTT \\
\hline$\ldots$ & G. A & $\ldots$ & . C & & & & $\ldots$ & $\ldots \mathrm{T}$. & $\ldots$ & $\ldots$ & $\ldots$ & $\ldots$ & & & $\ldots$ &.$T$ & C.. T. & ...A. \\
\hline$\ldots$ & . GC & $\ldots$ & & $\ldots$ & & & A... & . T. & $\cdots$ & AT. &. & $\ldots$ & & $\ldots$ & C.. & . & .T.T. & $\ldots$ \\
\hline$\ldots$ & AAC & $\ldots \ldots$ & & & & & G. & .. T. & & .AT. & $\cdots$ & $\cdots$ & & & & $\ldots$ & T.T. & $\cdots$ \\
\hline
\end{tabular}

M. raptor

M. raptorellus

M. zaraptor I

M. zaraptor II

$\begin{array}{ll}\text { M. raptor } & 24 \\ \text { M. raptorellus } 24\end{array}$

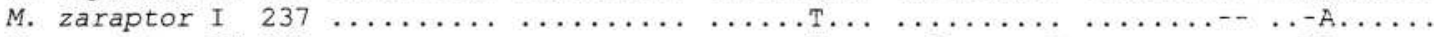

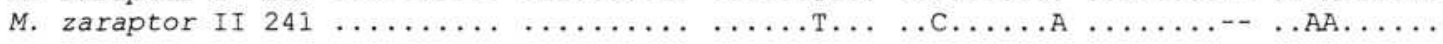

Fig. 3. Sequence comparison for Muscidifurax spp., represents the same amino acid or nucleotide is present as that in $M$. raptor; - , indicates a deletion. The $M$. raptorellus sequence represents both that species and $M$. uniraptor. Lower case letters in the $M$. raptor sequence represent primer sequences. 

M. raptor
M. raptorellus
M. zaraptor I
M. zaraptor II
M. raptor
$M$. raptorellus 301
M. zaraptor I 294
M. zaraptor II 299

M. raptor

$M$. raptorellus

M. zaraptor I

M. zaraptor II

M. raptor 361

M. raptorellus 361

M. zaraptor I 354

M. zaraptor II 359

M. raptor

M. raptorellus

M. zaraptor I

M. zaraptor II

M. raptor

$M$. raptorellus 421

M. zaraptor I 414

M. zaraptor II 419

\section{M. raptor}

M. raptorellus

M. zaraptor I

M. zaraptor II

M. raptor

M. raptorellus 4

M. zaraptor I 474

M. zaraptor II 479

\section{M. raptor}

M. raptorellus

M. zaraptor I

M. zaraptor II

M. raptor

M. raptorellus 541

M. zaraptor I 534

M. zaraptor II 539

\section{M. raptor}

M. raptorellus

M. zaraptor I

M. zaraptor II

M. raptor 601

M. raptorellus 601

M. zaraptor I 694

M. zaraptor II 699

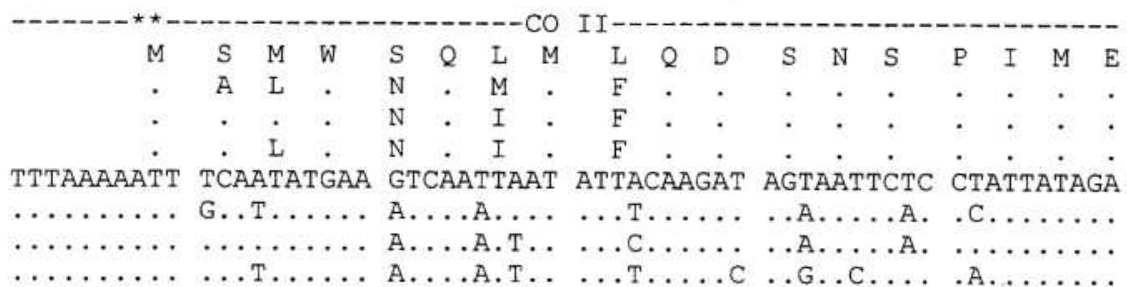

\begin{tabular}{|c|c|c|c|c|c|c|c|c|c|c|c|c|c|c|c|c|c|c|}
\hline M & M & I & $Y$ & $\mathrm{~F}$ & $\mathrm{H}$ & D & H & $S$ & M & M & V & I & M & V & I & I & $S$ & L \\
\hline$S$ & . & . & $M$ & . & . & . & . & G & . & L & I & . & I & M & . & . & . & M \\
\hline$S$ & . & . & $M$ & . & . & . & . & G & . & L & . & . & I & I & . & . & . & . \\
\hline$S$ & . & - & $\mathrm{M}$ & . & . & . & . & G & . & L & . & . & I & I & - & . & . & - \\
\hline
\end{tabular}

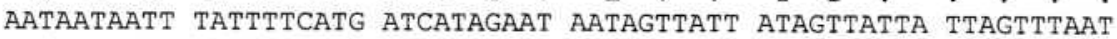

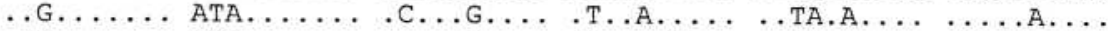

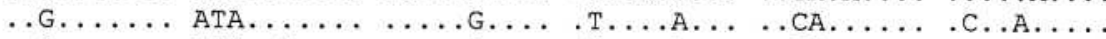

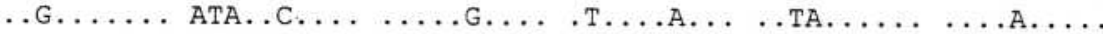

\begin{tabular}{|c|c|c|c|c|c|c|c|c|c|c|c|c|c|c|c|c|c|c|}
\hline M & Y & I & I & L & $\mathrm{F}$ & $M$ & F & $\mathrm{F}$ & $\mathrm{N}$ & $\mathrm{N}$ & L & $M$ & $\mathrm{~N}$ & $\mathrm{R}$ & F & M & L & $E$ \\
\hline L & . & . & . & $M$ & . & . & . & I & . & $\mathrm{T}$ & . & I & . & . & $\therefore$ & . & . & . \\
\hline L & . & . & . & $M$ & . & . & . & V & . & $\mathrm{T}$ & . & I & . & . & Y & . & . & . \\
\hline L & . & . & . & $M$ & . & . & . & $\mathrm{L}$ & . & $M$ & . & I & . & . & . & . & . & . \\
\hline
\end{tabular}

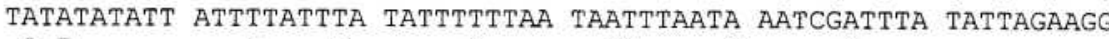

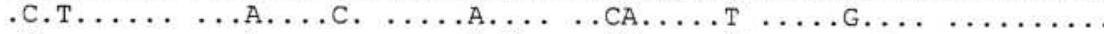

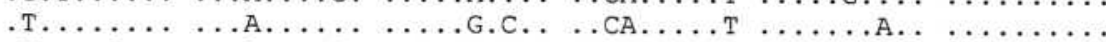

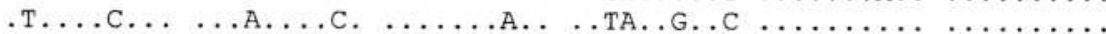

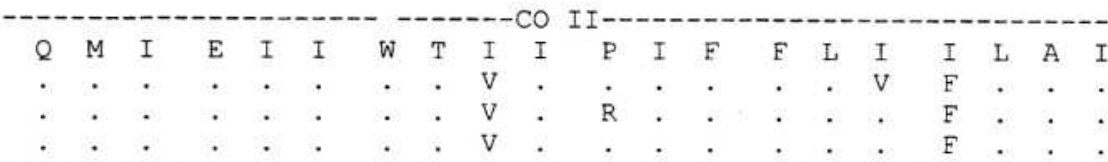
TCAAATAATT GAAATTATTT GAACAATTAT TCCAATTTT TTTTTAATCA TTTTAGCAAT

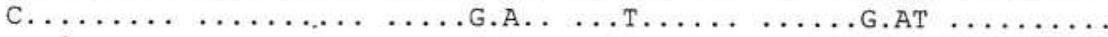

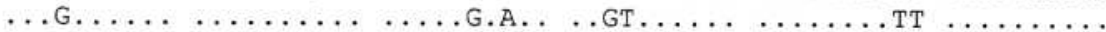

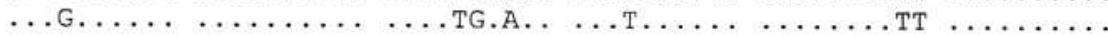

Fig. 3. Continued 


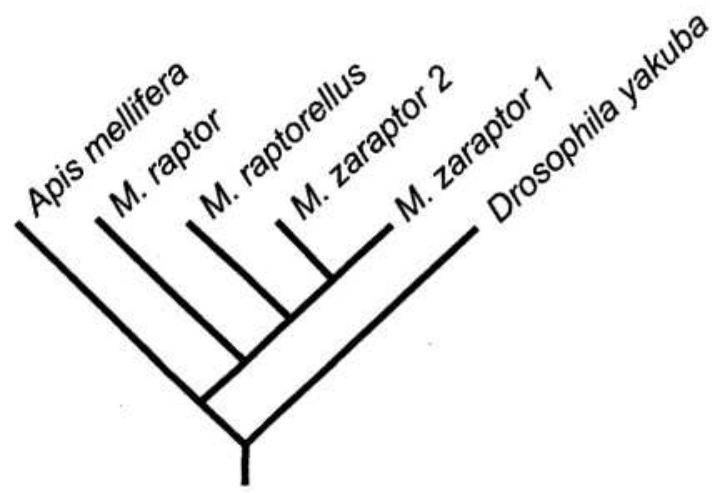

Fig. 4. Cladogram based upon sequence data of Muscidifurax spp., A. mellifera, and D. yakuba. All branches were supported by $\geq 97$ of 100 bootstrap replicates.

for $5 \mathrm{~min}$ with ethidium bromide $(1 \mu \mathrm{g} / \mathrm{ml})$. MetaPhor agarose (FMC Bioproducts, Rockland, ME ) gel electrophoresis was used to estimate fragment lengths. Gels (2.5\%) were run for $4 \mathrm{~h}$ in Gibco-BRL (Gaithersburg, MD) Horizon $11 \cdot 14$ gel boxes at $80 \mathrm{~V}$ with $1 \times$ TBE buffer. A 50-bp ladder (Gibco-BRL, Grand Island, NY) was used as a size standard. Ethidium bromide was added to the gel, $0.06 \mu \mathrm{g} / \mathrm{ml}$ final concentration, and $8 \mu \mathrm{g}$ of ethidium bromide was added to the buffer tray at the anodal end. Gels were interpreted on an ultraviolet $(312 \mathrm{~nm})$ transilluminator. Fragment sizes were calculated with the computer program GEL-JML (LaCroix 1994).

DNA Sequencing. Amplified DNA was purified using Geneclean II (Bio 101, Vista, CA) following manufacturer protocols and resuspended in $30 \mu \mathrm{l}$ of TE (pH 7.5). DNA was blunt-ended using New England Biolabs (Beverly, MA) reagents and ligated into pBluescript sk + plasmid using Stratagene (La Jolla, CA) reagents (Sambrook et al. 1989). Competent $E$. coli were transformed and positive colonies were verified by picking colonies with a sterile toothpick, replating and dipping the toothpick into a 50- $\mu$ l PCR reaction mixture (Sambrook et al. 1989). PCR reaction was performed as before but for only 20 cycles. Two $\mu$ l of PCR product was run on an agarose gel to determine if the insert was present. Positive clones were sequenced by the University of Nebraska-Lincoln Center for Biotechnology DNA Sequencing Laboratory (Lincoln, NE) using a LICOR Model 4000 DNA Sequencer (LI-COR, Lincoln, NE). Two primers, T3 and T7 promoters (Gibco-BRL, Gaithersburg, MD), were used for sequencing. Two clones were sequenced in both directions for each sample. Published sequences for $\boldsymbol{A}$. mellifera (Crozier and Crozier 1993) and D. yakuba (Clary and Wolstenholme 1985) were used for comparisons with those species.

Nucleotide sequences for $M$. raptor, $M$. raptorellus, and $M$. zaraptor (sequences 1 and 2) have been deposited in Genbank with accession numbers U97506-U97515.
Table 3. Nucleotide substitution (d) rates (below the diagonal) and transition to transversion ratios (above the diagnonal) among Muscidifurax spp., A. mellifera, and D. yakuba

\begin{tabular}{|c|c|c|c|c|c|c|}
\hline \multirow{2}{*}{ Species } & \multicolumn{6}{|c|}{ Species } \\
\hline & 1 & 2 & 3 & 4 & 5 & 6 \\
\hline 1 A. mellifera & - & 0.41 & 0.33 & 0.33 & 0.36 & 0.37 \\
\hline 2 D. yakuba & 0.53 & - & 0.27 & 0.31 & 0.28 & 0.32 \\
\hline $3 M$. raptor & 0.46 & 0.53 & - & 0.50 & 0.52 & 0.56 \\
\hline $4 M$. raptorellus & 0.53 & 0.61 & 0.19 & - & 0.66 & 0.82 \\
\hline $5 \mathrm{M}$. zaraptor 1 & 0.47 & 0.62 & 0.14 & 0.15 & - & 1.22 \\
\hline 6 M. zaraptor 2 & 0.54 & 0.60 & 0.17 & 0.18 & 0.08 & - \\
\hline
\end{tabular}

Statistical Analyses. Sequence divergence $(d)$ was calculated from restriction site data using The restriction enzyme analysis package (REAP) (McElroy et al. 1992) following the procedures of Nei and Tajima (1981) and Nei and Miller (1990). The DNADIST procedure of PHYLIP 3.5 (Felsenstein 1993) was used to calculate $d$ values from the sequence data using the Kimura 2-parameter model (Kimura 1980). The consensus dendrogram of the mtDNA haplotypes was derived using the Wagner parsimony method (DNAPARS and CONSENS procedures of PHYLIP). Bootstrap replicates (100) of the sequence data were generated with the SEQBOOT procedure of PHYLIP.

\section{Results}

The primer pair produced an amplicon estimated to be $632 \mathrm{bp}$ for all of the Muscidifurax spp. and C. macellaria with a $2.5 \%$ metaphor agarose gel (Fig. 1). The A. mellifera amplicon was estimated to be $856 \mathrm{bp}$.

Restriction Fragment Length Patterns. The restriction enzymes Alu I, Ava I, Ban II, Bfa I, Bsr I, Dde I, EcoR I, EcoR V, Hinc II, Hind III, Hpa I, Msp I, Pvu II, Rsa I, Sau96 I, Sac I, Scrf I, and Xba I did not cut the amplicon from any of the Muscidifurax samples. Twenty-four restriction sites were identified among the 4 Muscidifurax spp. for the remaining 9 restriction enzymes. The NY and NE samples of $M$. raptor gave identical restriction fragment patterns for all REs except Apo I. One of 2 wasps from the NY colony had an 81-bp band and lacked the 28-bp band. These bands were absent and present, respectively, in both of the NE and the other NY M. raptor (Fig. 2). The 3 samples of $M$. raptorellus and $M$. uniraptor gave identical restriction fragment patterns (Fig. 2; Table 2). Several of the restriction digests for $M$. zaraptor had faint bands in addition to the stronger bands, and the sum of the lengths of the bands consistently exceeded the length of the intact amplicon. Although these bands appeared to be caused by incomplete digests, they were not observed for the other species under similar digest conditions and could not be eliminated by increasing enzyme concentration or the duration of the digest. Because of these difficulties in interpreting the $M$. zaraptor restriction digests, this species was not included in the restriction site analysis. The 
Table 4. Restriction sites in Muscidifurax spp.

\begin{tabular}{|c|c|c|c|c|c|c|c|c|c|c|}
\hline \multirow{2}{*}{$\begin{array}{l}\text { Restriction } \\
\text { enzyme site }\end{array}$} & \multirow{2}{*}{ M. raptor } & \multirow{2}{*}{ M. raptorellus } & \multirow{2}{*}{ M. zaraptor $\mathbf{I}$} & \multirow{2}{*}{ M. zaraptor II } & \multicolumn{6}{|c|}{ M. zaraptor clones } \\
\hline & & & & & 1 & 2 & 3 & 4 & 5 & 6 \\
\hline \multicolumn{11}{|l|}{ Apo I } \\
\hline 183 & - & - & - & - & + & - & - & + & - & + \\
\hline 215 & + & + & + & + & + & + & + & + & + & + \\
\hline 224 & + & + & - & - & - & - & - & - & - & - \\
\hline 236 & - & + & - & - & - & - & - & - & - & - \\
\hline 270 & \pm & + & + & - & - & - & + & - & - & - \\
\hline 306 & + & - & + & + & + & + & + & + & + & + \\
\hline 343 & - & + & + & - & - & - & + & - & - & - \\
\hline 552 & + & + & + & + & - & + & + & + & + & - \\
\hline 577 & + & - & + & + & + & + & + & + & + & + \\
\hline 586 & - & + & - & - & - & - & - & - & - & - \\
\hline \multicolumn{11}{|l|}{ Ase I } \\
\hline 234 & - & - & + & + & - & + & + & - & + & + \\
\hline 326 & + & - & - & - & - & - & - & - & - & - \\
\hline 392 & - & + & - & - & - & - & - & - & - & - \\
\hline 415 & - & - & + & + & - & + & + & - & - & + \\
\hline 455 & - & + & + & - & ? & - & ? & ? & - & ? \\
\hline 458 & - & - & + & - & ? & - & ? & ? & - & ? \\
\hline \multicolumn{11}{|l|}{ DdeI } \\
\hline 60 & - & - & - & + & - & + & - & - & + & + \\
\hline Dpn II & & & & & & & & & & \\
\hline 379 & + & - & + & + & + & + & + & + & + & + \\
\hline 455 & - & - & - & + & - & + & - & - & + & - \\
\hline Dra I & & & & & & & & & & \\
\hline 242 & + & + & + & + & + & + & + & + & + & + \\
\hline 269 & - & + & + & + & + & + & + & + & + & + \\
\hline 303 & + & + & + & + & + & + & + & + & + & + \\
\hline 446 & - & - & - & + & + & + & - & + & + & + \\
\hline 549 & - & + & - & - & + & - & - & - & - & + \\
\hline Hae III & & & & & & & & & & \\
\hline 480 & - & + & - & - & - & - & - & - & - & - \\
\hline $\operatorname{Hinf} \mathrm{I}$ & & & & & & & & & & \\
\hline 28 & + & + & + & + & + & + & + & + & + & + \\
\hline 155 & - & + & - & - & - & - & - & - & - & - \\
\hline 176 & - & + & - & - & - & - & - & - & - & - \\
\hline 552 & - & - & - & - & + & - & - & - & - & + \\
\hline 571 & - & - & + & - & - & - & + & - & - & - \\
\hline Mse I & & & & & & & & & & \\
\hline 146 & + & + & + & + & & & & & & \\
\hline 209 & - & - & + & + & & & & & & \\
\hline 234 & - & + & + & + & & & & & & \\
\hline 241 & + & + & + & + & & & & & & \\
\hline 268 & + & + & + & + & & & & & & \\
\hline 289 & - & + & - & - & & & & & & \\
\hline 302 & + & + & + & + & & & & & & \\
\hline 326 & + & - & - & - & & & & & & \\
\hline 392 & - & + & - & - & & & & & & \\
\hline 416 & + & - & + & + & & & & & & \\
\hline 447 & + & - & - & + & & & & & & \\
\hline 455 & + & + & + & - & & & & & & \\
\hline 458 & - & + & + & - & & & & & & \\
\hline 524 & + & - & + & + & & & & & & \\
\hline 548 & + & - & + & + & . & & & & & \\
\hline 562 & - & - & + & + & & & & & & \\
\hline 575 & - & + & - & - & & & & & & \\
\hline 590 & - & + & - & + & & & & & & \\
\hline 597 & + & - & + & + & & & & & & \\
\hline Ssp I & & & & & & & & & & \\
\hline 92 & + & - & - & - & - & - & - & - & - & - \\
\hline 287 & - & + & - & - & + & - & - & + & - & - \\
\hline 330 & + & + & - & - & - & - & - & - & - & - \\
\hline 390 & - & + & + & + & - & + & + & + & + & + \\
\hline 580 & - & + & - & - & - & - & - & - & - & - \\
\hline Taq I & & & & & & & & & & \\
\hline 6 & + & + & + & + & + & + & + & + & + & + \\
\hline 129 & - & - & + & - & - & - & + & - & - & - \\
\hline 170 & - & - & - & - & + & - & - & - & - & - \\
\hline 463 & + & - & + & + & + & + & + & + & + & + \\
\hline
\end{tabular}

Site positions are based on $M$. raptor map (Fig. 3). M. uniraptor was identical to $M$. raptorellus for all restriction sites. Site presence $(+)$ and absence $(-)$ were based on the sequences and verified by RFLP analysis for $M$, raptor, $M$. raptorellus, $M$. uniraptor, and $M$. zaraptor types I and II. Data for clones 1-6 of M. zaraptor are based on RFLP data only. 
Table 5. Restrietion fragment lengths estimated on $2.5 \%$ MetaPhor agarose gels for 6 M. aaraptor clones

\begin{tabular}{|c|c|c|c|c|c|c|}
\hline $\begin{array}{l}\text { Restriction } \\
\text { enzymes }\end{array}$ & Clone 1 & Clone 2 & Clone 3 & Clone 4 & Clone 5 & Clone 6 \\
\hline Apo I & $271,186,95,50$ & $245,216,95,50$ & $214,214,62,53$ & $247,189,99,51$ & $247,219,98,51$ & $271,221,98,51$ \\
\hline Ase I & 456,170 & $231,209,184$ & $229,184,170$ & 443,168 & 377,234 & $234,184,168$ \\
\hline Dde I & 617 & 532,66 & 617 & 617 & 536,66 & 543,66 \\
\hline Dpn II & 377,246 & $373,170,84$ & 368,246 & 373,243 & $373,168,83$ & 371,243 \\
\hline Dra I & $245,146,106,78,60$ & $243,180,146,33^{a}$ & $321,240,33^{a}$ & $240,180,146,34^{a}$ & $240,177,146,34^{a}$ & $240,146,105,81,60$ \\
\hline $\operatorname{Hinf} \mathbf{I}$ & 525,76 & 571 & 518,56 & 576 & 576 & 511,76 \\
\hline Mse I & $149^{a}, 67^{a}$ & $149,117,82,67$ & $145,117,68^{a}$ & $149^{a}, 68^{a}$ & $150,{ }^{a}, 82,68$ & $151,119,68$ \\
\hline Ssp I & 330,291 & 381,236 & 368,234 & $284,231,99$ & 281,234 & 372,234 \\
\hline Taq I & $293,164^{a}$ & 442,160 & $325,162,122$ & 447,160 & 447,160 & 447,160 \\
\hline
\end{tabular}

${ }^{a}$ Possibly double bands.

estimated number of nucleotide substitutions per nucleotide, $d$, calculated from restriction site data (Nei and Tajima 1981) was 0.23 between $M$. raptor and $M$. raptorellus-M. uniraptor. All 9 restriction enzymes gave restriction patterns that were diagnostic between $M$. raptor and $M$. raptorellus $-M$. uniraptor.

Sequence Analysis. Sequencing the amplicon revealed that it was 625 bp long for $M$. raptor, $M$. raptorellus, and $M$. uniraptor (Fig. 3). Sequences for Nebraska and New York $M$. raptor samples were identical except for nucleotide bp 273 (Fig. 3), which exhibited a $\mathrm{T}$ to $\mathrm{G}$ transversion in 1 of the 2
M. raptor from New York. Sequences for Chile, Peru, and Nebraska $M$. raptorellus and $M$. uniraptor were identical. Two distinct sequences were obtained from 2 clones of $M$. zaraptor: clone 1 was 618 bp long and clone 2 was $623 \mathrm{bp}$. The 4 -bp deletion observed in clone 1 (bps 99-102 in Fig. 4) imparted a frame shift in the COI gene. This frame shift changed 12 amino acids before reaching a stop codon and truncating the final 31 amino acids of the subunit. $A$. mellifera differed from D. yakuba and the Muscidifurax spp. by a 30 -bp insert at the $3^{\prime}$ end of the COI gene (after base 234 of the $M$. raptor sequence) and a 192-bp A + T-rich insert between tRNA ${ }^{\text {Leu }}$ and

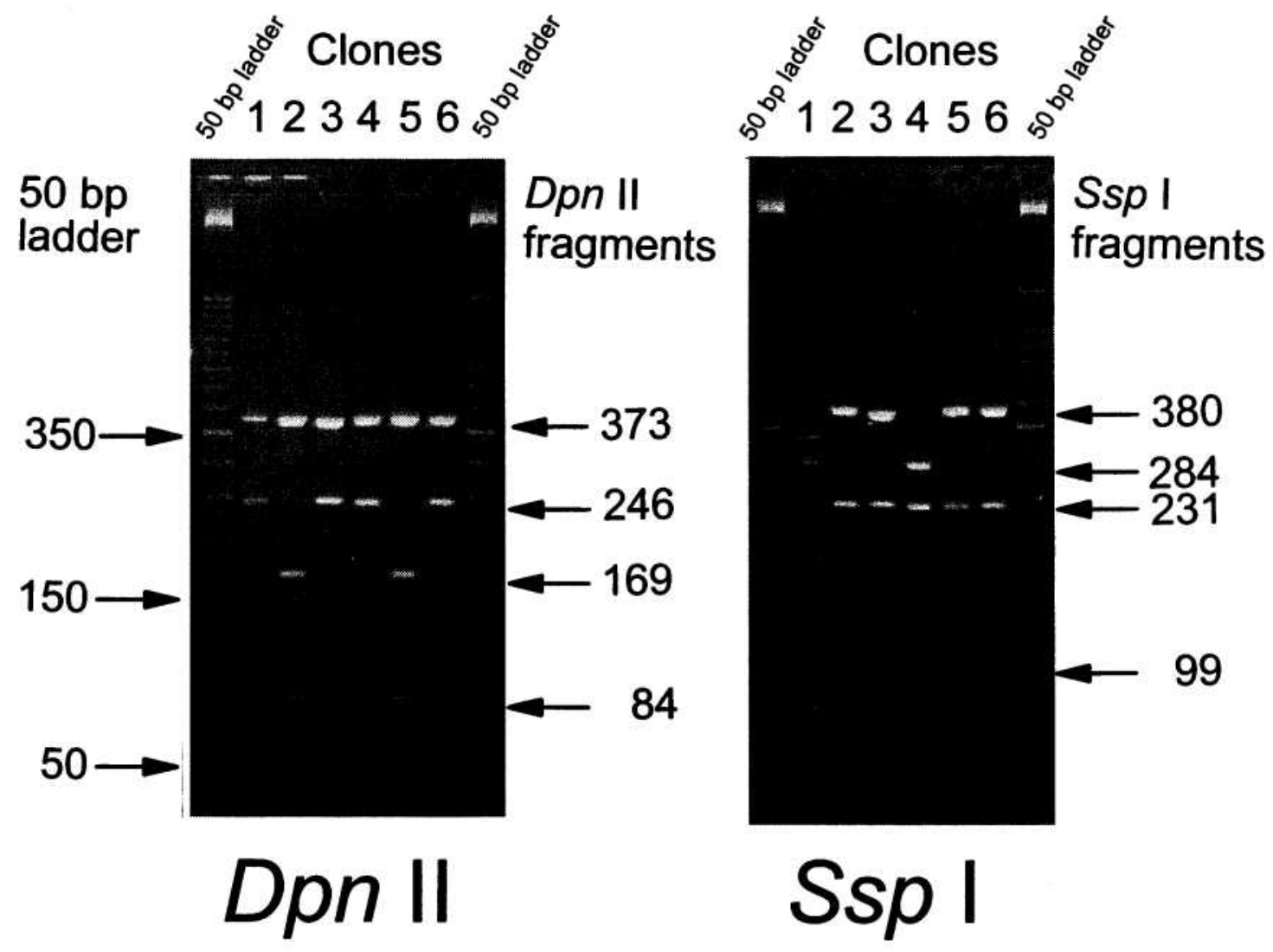

Fig. 5. Dpn II and Ssp I digests of amplicon from $6 \mathrm{M}$. zaraptor clones on $2.5 \%$ MetaPhor agarose gels. 
COII (after base 307 of the $M$. raptor sequence) (Fig. 3). These inserts were ignored in the comparisons with A. mellifera. Muscidifurax spp. differed from A. mellifera by 0.50 nucleotide substitutions per site and from $D$. yakuba by 0.59 substitutions per site. Divergence among the Muscidifurax spp. ranged between 0.14 and 0.19 substitutions per site (Table 3 ). The $2 M$. zaraptor sequences differed by 0.08 substitutions per site. Transition to transversion ratios ranged between 0.50 and 1.22 among the Muscidifurax spp., with the lower values being associated with higher $d$ values (Table 3 ). The $A+T$ content of the amplicon was $81-84 \%$ in the Muscidifurax sp. compared with 75 and $84 \%$ in D. yakuba and $A$. mellifera, respectively.

Parsimony analysis resulted in a single most parsimonious cladogram with 467 state changes (Fig. 4). The $2 M$. zaraptor sequences clustered together. All branches of the cladogram were supported by $\geq 97$ of 100 bootstrap replicates of the dataset.

Sequences were scanned for restriction enzyme sites with the computer program DIGEST (written by Ramin Nakisa). All of the restriction patterns observed in the RFLP analysis were supported by restriction enzyme sites in the sequences (Table 4).

The $M$. zaraptor colony used for the study described study was discarded before realizing that it contained distinct mitochondrial haplotypes. In an effort to see if both haplotypes were present in the new $M$. zaraptor colony, amplification products from the new colony were cloned as for sequencing. DNA from individual clones was amplified by PCR, and amplicons from the 6 positive clones were digested with 9 restriction enzymes (Table 5). These enzymes were chosen because the sequence data indicated they were diagnostic between the $2 \mathrm{M}$. zaraptor sequences. Each of the 6 clones resulted in a unique composite digest pattern (Fig. 5). Two of the patterns, clones 3 and 2 , matched the digest patterns predicted for the $M$. zaraptor I and II sequences. Restriction site analysis indicated that $d$ values ranged from 0.03 to 0.10 among the clones.

To isolate $M$. zaraptor lines with each of the observed haplotypes for morphological comparisons and further genetic studies, we initiated 10 isofemale lines from the $M$. zaraptor colony. DNA was isolated from $F_{1}$ wasps (5 wasps pooled and individual wasps) from each line, amplified, and digested with the diagnostic restriction enzymes Apo I, Ase I, Dde I, Dpn II, Hinf I, Mse I, Ssp I, and Taq I. Restriction digest patterns were identical for the pooled and individual DNA isolations, although amplifications were stronger from the pooled samples. Banding patterns were identical for 9 of the 10 lines and the same as those observed with the earlier pooled samples from the discarded colony (Fig. 2). The sum of the estimated lengths of the observed bands greatly exceeded the length of the amplicon, and the banding patterns appeared to be composites of the patterns observed in the 6 clones combined. The banding pattern of the 10th line lacked several of the bands observed in the other 9 , but no new bands were observed.

\section{Discussion}

The estimated length of the amplicons was close to that predicted by the $D$. yakuba sequence (Clary and Wolstenholme 1985). The 193-bp A + T-rich, noncoding insert found between the RNA $^{\text {leu }}$ and COII genes by Crozier and Crozier (1993) in A. mellifera was not present in Muscidifurax sp. As in other non-Apis hymenopterans (Cornuet et al. 1991), no nucleotides are present between the tRNA $^{\text {leu }}$ and COII genes.

Four restriction enzymes, Apo I, Ase I, Mse I, and Ssp I, resulted in digest patterns which were diagnostic among $M$. raptor, $M$. raptorellus $-M$. uniraptor, and $M$. zaraptor. An additional 5 enzymes, Dpn II, Dra I, Hae III, Hinf I, and Taq I, differentiated $M$. raptorellus-M. uniraptor from the other species. RFLP analysis could not differentiate the geographic isolates of $M$. raptor or $M$. raptorellus nor could they separate $M$. raptorellus from $M$. uniraptor. Use of PCR-RFLP patterns for species diagnostics of Muscidifurax spp. must be used with caution until the questions regarding the multiple, divergent sequences in $M$. zaraptor is resolved.

The sequencing analysis supported the variation detected by PCR-RFLP analysis. Predicted restriction sites were observed in all sequences to account for the observed RFLP patterns. Although a single polymorphic nucleotide sight was observed in the New York sample of $M$. raptor, no fixed differences were observed among the sequences from the geographic isolates of $M$. raptor and $M$. raptorellus. The $M$. uniraptor sequence was identical to that of $M$. raptorellus. These data support the characterization of the Nebraska isolate of $M$. raptorellus as being true to that species, with the probable origin being an introduction from South America (Antolin et al. 1996).

The status of $M$. uniraptor is not resolved. The mtDNA amplicon sequence for this species was identical to that of $M$. raptorellus. Kogan and Legner (1970) indicated that $M$. uniraptor was "extremely difficult" to distinguish from $M$. raptor and "almost impossible" to differentiate from $M$. raptoroides. However, a fringe of setae on the margins of $M$. uniraptor wings "easily" distinguish it from M. raptorellus and M. zaraptor. Fringe setae were apparent on the wings of M. uniraptor from our colony. Legner (1969) indicated that $M$. uniraptor males, on occasion, were able to fertilize $M$. raptor females successfully, but he did not observe successful hybridization between $M$. uniraptor and $M$. raptorellus. Based upon further hybridization data, Legner (1987) suggested "the evolution of $M$. uniraptor from M. raptor." Isozyme data (Kawooya 1983) appear to support the relationship between $M$. uniraptor and $M$. raptor. However, extremely high, withinspecies genetic distances (Nei [1978] unbiased genetic distances of $0.40,0.50$, and 0.76 for $M$. raptor 
from North Carolina, Utah, and Israel) were observed in that study. These distances are far larger than expected for within-species variation (Ayala et al. 1974), making Kawooya's results difficult to interpret. Our mtDNA data clearly indicate that $M$. uniraptor is more closely related to $M$. raptorellus than to $M$. raptor.

Currently, our data are insufficient to evaluate the specific status of $M$. uniraptor. Thelytokous reproduction was the primary characteristic used by Kogan and Legner (1970) to distinguish this species. Subsequent studies have implicated Wolbachia, a cytoplasmically inherited microorganism, as the cause for thelytokous reproduction in $M$. uniraptor (Stouthamer et al. 1993). Wolbachia infections usually can be eliminated with antibiotics or high temperatures (Stouthamer and Luck 1988), rendering thelytokous strains arrhenotokous. Crosses between Wolbachia-free $M$. uniraptor and M. raptorellus will be needed to confirm the specific status of $M$. uniraptor (Stouthamer et al. 1990).

The levels of sequence divergence observed among $M$. raptor, $M$. raptorellus, and $M$. zaraptor were higher than expected. Dowton and Austin (1995) indicate that a higher rate of mtDNA divergence may be associated with parasitism in Hymenoptera. Nevertheless, the levels of divergence observed between the species indicate divergence times of several millions of years (Powell et al. 1986); not the $400 \mathrm{yr}$ required if Muscidifurax were of Old World origin. These data indicate that the presence of Muscidifurax in the New World predates the arrival of house flies and stable flies, their preferred hosts. The phylogeographic structure of the group in the Americas adds further support to this argument. One question which remains to be resolved is whether $M$. raptor was a Holarctic species, or if its introduction to the Old World was a recent event.

Our data for $M$. zaraptor raise several questions. Digests from progeny of individual females indicate that multiple haplotypes are present within each line and within individual wasps. Hence, the mtDNA variation observed in $M$. zaraptor is caused by variation within individuals rather than population differentiation indicative of multiple cryptic species. Further complicating the issue is the frame shift deletion in the $M$. zaraptor 1 sequence. We must assume that a COI subunit with the C-terminal 43 amino acids modified or truncated is nonfunctional. Two explanations for the multiple haplotypes are as follows: (1) the species has a high level of heteroplasmy with extremely high levels of differentiation among the mtDNA haplotypes, or (2) a portion of the mitochondrial genome has been translocated to the nuclear genome where it has formed tandem repeats (Lopez et al. 1994, Zhang and Hewitt 1996a). Heteroplasmy has been reported for several insect species (Boyce et al. 1989, 1994; Harrison 1989). However, in nearly all cases, the heteroplasmy has been the result of length variation in the $\mathbf{A}+\mathrm{T}$-rich region, not substitutions in the coding regions. Recent translocation and amplification of mitochondrial sequences in the nuclear genome has been reported for the desert locust (Zhang and Hewitt 1996b). In future studies, the variant digest pattern observed in the exceptional $M$. zaraptor isofemale line will be useful for determining the mode of inheritance.

\section{Acknowledgments}

We thank Wes Watson, J. H. Werren, and M. D. Ellis for samples of $M$. raptor, $M$. uniraptor, and A. mellifera, respectively, and T. O. Powers for permitting us to do some aspects of this work in his laboratory. T. O. Powers, K. P. Pruess, R. L. Roehrdanz, and B. D. Siegfried provided helpful suggestions and critical reviews of the manuscript. This work was done in cooperation with the Institute of Agriculture and Natural Resources, University of Nebraska, Lincoln, and is published as Journal Series, Nebraska Agricultural Research Division Paper 11709.

\section{References Cited}

Antolin, M. F., D. S. Guertin, and J. J. Petersen. 1996. The origin of gregarious Muscidifurax (Hymenoptera: Pteromalidae) in North America: an analysis using molecular markers. Biol. Control 6: 76-82.

Ayala, F. J., M. L. Tracey, D. Hedgecock, and R. C. Richmond. 1974. Genetic differentiation during the speciation process in Drosophila. Evolution 28: 576-592.

Bogdanowicz, S. M., W. E. Wallner, J. Bell, T. M. Odell, and R. G. Harrison. 1993. Asian gypsy moths (Lepidoptera: Lymantriidae) in North America: evidence from molecular data. Ann. Entomol. Soc. Am. 86: 710715 .

Boyce, T. M., M. E. Zwick, and C. F. Aquadro. 1989. Mitochondrial DNA in the bark weevils: size, structure and heteroplasmy. Genetics 123: 825-836.

1994. Mitochondrial DNA in the bark weevils: phylogeny and evolution in the Pissodes strobi species group (Coleoptera: Curculionidae). Mol. Biol. Evol. 11: 183194.

Clary, D. O., and D. R. Wolstenholme. 1985. The mitochondrial DNA molecule of Drosophila yakuba: nucleotide sequence, gene organization, and genetic code. J. Mol. Evol. 22: 252-271.

Cornuet, J.-M., L. Garnery, and M. Solignac. 1991. Putative origin and function of the intergenic region between COI and COII of Apis mellifera L. mitochondrial DNA. Genetics 128: 393-403.

Crozier, R. H., and Y. C. Crozier. 1993. The mitochondrial genome of the honeybee Apis mellifera: complete sequence and genome organization. Genetics 133: 97117.

Dowton, M., and A. D. Austin. 1995. Increased genetic diversity in mitochondrial genes is correlated with the evolution of parasitism in the Hymenoptera. J. Mol. Evol. 41: 958-965.

Felsenstein, J. 1993. PHYLIP: Phylogeny inference package, version 3.5. The University of Washington, Seattle.

Harrison, R. G. 1989. Animal mitochondrial DNA as a genetic marker in population and evolutionary biology. Trends Ecol. Evol. 4: 6-11.

Kawooya, J. K. 1983. Electrophoretic discrimination of species of the Muscidifurax (Hymenoptera: Pteroma- 
lidae) complex. Ph.D. dissertation, University of Illinois, Urbana.

Kimura, M. 1980. A simple model for estimating evolutionary rates of base substitutions through comparative studies of nucleotide sequences. J. Mol. Evol. 16: 111-120.

Kogan, M., and E. F. Legner. 1970. A biosystematic revision of the genus Muscidifurax (Hymenoptera: Pteromalidae) with descriptions of four new species. Can. Entomol. 102: 1268-1290.

LaCroix, J. M. 1994. DNA fragment size determination on agarose gel by using the application GEL. Comp. Appl. Biosci. 10: 185-187.

Legner, E. F. 1969. Reproductive isolation and size variation in the Muscidifurax raptor complex. Ann. Entomol. Soc. Am. 62: 382-385.

1983. Broadened view of Muscidifurax parasites associated with endophilous synanthropic flies and sibling species in the Spalangia endius complex, pp. 47-48. In C. D. Grand [ed.], Proceedings and papers of the 51st Annual Conference of the Calififornia Mosquito and Vector Control Association. San Mateo, CA, 23-26 January 1983. CMVCA, Sacramento, CA.

1987. Transfer of thelytoky to arrhenotokous Muscidifurax raptor Girault and Sanders (Hymenoptera: Pteromalidae). Can. Entomol. 119: 265-271.

1988. Hybridization in principal parasitoids of synanthropic Diptera: the genus Muscidifurax (Hymenoptera: Pteromalidae). Hilgardia 56: 1-36.

Lopez, J. V., N. Yuhki, R. Masuda, W. Modi, and S. J. O'Brien. 1994. Numt, a recent transfer and tandem amplification of mitochondrial DNA to the nuclear genome of the domestic cat. J. Mol. Evol. 39: 174-190.

Lysyk, T. J. 1995. Parasitoids (Hymenoptera: Pteromalidae, Ichneumonidae) of filth fly (Diptera: Muscidae) pupae at dairies in Alberta. J. Econ. Entomol. 88: 659665.

McElroy, D., P. Moran, E. Bermingham, and I. Kornfield. 1992. REAP: an integrated environment for the manipulation and phylogenetic analysis of restriction data. J. Hered. 83: 157-158.

Miller, R. W., and D. A. Rutz. 1990. Survey of house fly pupal parasitoids on dairy farms in Maryland and New York, pp. 59-67. In D. A. Rutz and R. S. Patterson [eds.], Biocontrol of arthropods affecting livestock and poultry. Westview, Boulder, CO.

Nei, M. 1978. Estimation of average heterozygosity and genetic distance from a small number of individuals. Genetics 89: 583-590.

Nei, M., and J. C. Miller. 1990. A simple method for estimating average number of nucleotide substitutions within and between populations from restriction data. Genetics 125: 873-879.

Nei, M., and F. Tajima. 1981. DNA polymorphism detectable by restriction endonucleases. Genetics 97 : 145-163.
Petersen, J. J., and J. R. Matthews. 1984. Effects of freezing of host pupae on the production of progeny by the filth fly parasite Muscidifurax zaraptor (Hymenoptera: Pteromalidae). J. Kans. Entomol. Soc. 57: 387-393.

Petersen, J. J., D. W. Watson, and B. M. Pawson. 1990. Native biocontrol agents as a component of integrated pest management for confined livestock, pp. 79-93. In D. A. Rutz and R. S. Patterson [eds.], Biocontrol of arthropods affecting livestock and poultry. Westview, Boulder, CO.

Powell, J. R., A. Caccone, G. D. Amato, and C. Yoon. 1986. Rates of nucleotide substitution in Drosophila mitochondrial DNA and nuclear DNA are similar. Proc. Natl. Acad. Sci. U.S.A. 83: 9090-9093.

Propp, G. D. 1986. Characterization of intra- and interspecific isoenzyme variation in Spalangia spp. and Muscidifurax spp. (Chalcidoidea: Pteromalidae): parasitoids of synanthropic diptera, pp. 164-174. In R. S. Patterson and D. A. Rutz [eds.], Biological control of muscoid flies. Misc. Publ. Entomol. Soc. Am. 61.

Sambrook, J., E. F. Fritsch, and T. Maniatis. 1989. Molecular cloning: a laboratory manual, 2nd ed. Cold Spring Harbor Laboratory Press, NY.

Stouthamer, R., and R. F. Luck. 1988. Micro-organisms implicated as a cause of thelytokous parthenogenesis in Trichogramma spp. (Hymenoptera: Trichogrammatidae), p. 256. In Proceedings of the XVIII International Congress of Entomology. Vancouver, B.C., Canada, 3-9 July 1988.

Stouthamer, R., J. D. Pinto, G. R. Platner, and R. F. Luck. 1990. Taxonomic status of thelytokous forms of $T r i$ chogramma (Hymenoptera: Trichogrammatidae). Ann. Entomol. Soc. Am. 83: 475-481.

Stouthamer, R., J.A.J. Breeuwer, R. F. Luck, and J. H. Werren. 1993. Molecular identification of microorganisms associated with parthenogenesis. Nature (Lond.) 61: 66-68.

Taylor, D. B., A. L. Szalanski, and R. D. Peterson II. 1996. Identification of screwworm species by polymerase chain reaction-restriction fragment length polymorphism. Med. Vet. Entomol. 10: 63-70.

Zhang, D. X., and G. M. Hewitt. 1996a. Nuclear integrations: challenges for mitochondrial DNA markers. Trends Ecol. Evol. 11: 247-251.

1996b. Highly conserved nuclear copies of the mitochondrial control region in the desert locust Schistocerca gregaria: some implications for population studies. Mol. Ecol. 5: 295-300.

Received for publication 20 January 1997; accepted 12 May 1997. 